\title{
Low Energy Antikaon-nucleon/nuclei interaction studies by AMADEUS
}

\author{
K. Piscicchia ${ }^{1,2, *}$, M. Bazzi ${ }^{2}$, G. Belloti ${ }^{3}$, A. M. Bragadireanu ${ }^{4}$, D. Bosnar ${ }^{5}$, A. D. Butt ${ }^{3,6}$, \\ M. Cargnelli ${ }^{7}$, C. Curceanu ${ }^{2}$, R. Del Grande ${ }^{2}$, L. De Paolis ${ }^{2,8}$, L. Fabbietti ${ }^{9}, 10$, C. Fiorini ${ }^{3,6}$, \\ F. Ghio ${ }^{11,12}$, C. Guaraldo ${ }^{2}$, R. S. Hayano ${ }^{13}$, M. Iliescu ${ }^{2}$, M. Iwasaki ${ }^{14}$, P. Levi Sandri ${ }^{2}$,

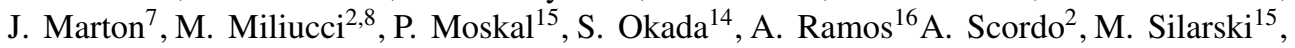 \\ D. L. Sirghi ${ }^{2,4}$, F. Sirghi ${ }^{2,4}$, M. Skurzok ${ }^{15}$, A. Spallone ${ }^{2}$, O. Vazquez Doce ${ }^{9,10}$, \\ E. Widmann ${ }^{7}$, S. Wycech ${ }^{17}$, and J. Zmeskal ${ }^{7}$
}

${ }^{1}$ CENTRO FERMI - Museo Storico della Fisica e Centro Studi e Ricerche "Enrico Fermi", Roma, Italy

${ }^{2}$ INFN Laboratori Nazionali di Frascati, Frascati, Rome, Italy

${ }^{3}$ Politecnico di Milano, Dip. di Elettronica, Informazione e Bioingegneria, Milano, Italy

${ }^{4}$ Horia Hulubei National Institute of Physics and Nuclear Engineering, Magurele, Romania

${ }^{5}$ University of Zagreb, Zagreb, Croatia

${ }^{6}$ INFN Sezione di Milano, Milano, Italy

${ }^{7}$ Stefan-Meyer-Institut für Subatomare Physik, Wien, Austria

${ }^{8}$ Università degli Studi di Roma Tor Vergata, Rome, Italy

${ }^{9}$ Excellence Cluster 'Origin and Structure of the Universe', Garching, Germany

${ }^{10}$ Physik Department E12, Technische Universität München, Garching, Germany

${ }^{11}$ INFN Sezione di Roma I, Rome, Italy

${ }^{12}$ Istituto Superiore di Sanità, Rome, Italy

${ }^{13}$ The University of Tokyo, Tokyo, Japan

${ }^{14}$ RIKEN, The Institute of Physics and Chemical Research, Saitama, Japan

${ }^{15}$ Institute of Physics, Jagiellonian University, Kracow, Poland

${ }^{16}$ Departament de Fisica Quantica i Astrofisica and Institut de Ciencies del Cosmos, Universitat de Barcelona, Barcelona, Spain

${ }^{17}$ National Centre for Nuclear Research, Warsaw, Poland

\begin{abstract}
The AMADEUS experiment at the DAФNE collider of LNF-INFN deals with the investigation of the at-rest, or low-momentum, $\mathrm{K}^{-}$interactions in light nuclear targets, with the aim to constrain the low energy QCD models in the strangeness sector. The 0 step of the experiment consisted in the reanalysis of the 2004/2005 KLOE data, exploiting $\mathrm{K}^{-}$absorptions in $\mathrm{H},{ }^{4} \mathrm{He},{ }^{9} \mathrm{Be}$ and ${ }^{12} \mathrm{C}$, leading to the first invariant mass spectroscopic study with very low momentum (about $100 \mathrm{MeV}$ ) in-flight $\mathrm{K}^{-}$captures. With AMADEUS step 1 a dedicated pure Carbon target was implemented in the central region of the KLOE detector, providing a high statistic sample of pure at-rest $\mathrm{K}^{-}$nuclear interaction. The first measurement of the non-resonant transition amplitude $\left|A_{K^{-} n \rightarrow \Lambda \pi^{-}}\right|$at $\sqrt{s}=33 \mathrm{MeV}$ below the $\overline{\mathrm{K}} \mathrm{N}$ threshold is presented, in relation with the $\Lambda(1405)$ properties studies. The analysis procedure adopted in the serarch for $\mathrm{K}^{-}$multinucleon absorption cross sections and Branching Ratios will be also described.
\end{abstract}

\footnotetext{
*e-mail: kristian.piscicchia@lnf.infn.it
} 


\section{Introduction}

The absorption of low momentum $\mathrm{K}^{-}$mesons $\left(p_{K} \sim 127 \mathrm{MeV} / \mathrm{c}\right)$, produced by the DAФNE [1] collider, on He and $\mathrm{C}$ nuclear targets is investigated, with the aim to get information on the resonant and non-resonant transition amplitudes few $\mathrm{MeV}$ below the $\overline{\mathrm{K}} \mathrm{N}$ threshold, these represent good tests for the theoretical predictions of the low energy QCD models in the strangeness sector.

The detailed characterisation of the yield and spectral shape of the non-resonant antikaonnucleon absorption, resulting in a hyperon-pion final state below the $\overline{\mathrm{K}} \mathrm{N}$ threshold, is also a fundamental reference to extract the $\Lambda(1405)$ (isospin $I=0$ ) properties in $\overline{\mathrm{K}} \mathrm{N}$ absorption experiments from the competing non-resonant $(\Sigma \pi)^{0}$ formation. According to the chiral unitary model [2-4] (see also the review [5]) the $\Lambda(1405)$ can be interpreted as a molecular mesonbaryon state, emerging from the interference of two poles, a lower mass pole (about 1390 $\mathrm{MeV}$ ) which is mainly coupled to the $\Sigma \pi$ channel and a high mass pole, coupled to the $\overline{\mathrm{K}} \mathrm{N}$ production channel, located around $1420 \mathrm{MeV}$ (the next to leading order calculation of the poles masses and widths can be found in [6]). For this reason the shape of the measured $(\Sigma \pi)^{0}$ invariant mass distribution is expected to change depending on the decay channel, due to the isospin interference term (which contributes with opposite sign to the $\Sigma^{ \pm} \pi^{\mp}$ cross sections, and vanishes for $\Sigma^{0} \pi^{0}$ ) but also on the production channel. In this scenario the $\overline{\mathrm{K}} \mathrm{N}$ absorption represents the golden channel for investigating the predicted high mass pole of the $\Lambda(1405)$.

The strength of the $\overline{\mathrm{K}} \mathrm{N}$ sub-threshold interaction potential also determines the possibility for the formation of $\overline{\mathrm{K}}$ multi-nucleon bound states. It is well known from the experimental search of such exotic states in kaon induced reactions that they overlap with the multi-nucleon absorption processes over a broad range of the phase space (see for example Ref. [7-10]). Consequently the precise determination of the $\mathrm{K}^{-}$multi-nucleon absorption reactions yields is of great importance. Also, in heavy-ion collision the exclusive measurement of the lowenergy cross sections of antikaons off nuclei is crucial to constraint the theoretical interpretation of the data [11]. The analysis procedure adopted in the search for $\mathrm{K}^{-}$multi-nucleon absorption cross sections and Branching Ratios (BR) will be described.

The analysed data sample, collected by the KLOE collaboration in 2004/2005 [12], allows to investigate both at rest $\left(p_{K} \sim 0\right)$ and in-flight low momentum $\mathrm{K}^{-}$nuclear absorptions. In the case of interactions at-rest the underlying mechanism consists in the capture of the strange meson in a highly excited atomic state and a successive cascade to low-lying states, followed by the $\mathrm{K}^{-}$nuclear capture. Besides the interactions at-rest, an important contribution from in-flight $\mathrm{K}^{-}$nuclear captures was already characterised by the AMADEUS collaboration in previous works $[13,14]$. The in-flight capture process consists in the kaon propagation through the electron cloud, followed by the nuclear absorption. We will show how an insight on the $\Lambda(1405)$ high mass pole characteristic can be obtained from the in-flight $\mathrm{K}^{-}$absorption reactions on $\mathrm{He}$ and $\mathrm{C}$.

\section{Data samples}

The ongoing AMADEUS analyses refer to two data samples. One is represented by the data collected by the KLOE collaboration during the 2004/2005 data taking, corresponding to $1.74 \mathrm{fb}^{-1}$. The KLOE detector is used as an active target, the hadronic interaction of negative kaons with the materials of the apparatus being investigated; in particular $\mathrm{K}^{-9} \mathrm{Be}$ absorptions in the DA $\Phi N E$ Beryllium thin cylindrical layer and the DAФNE aluminated beryllium pipe, $\mathrm{K}^{-12} \mathrm{C}$ and $\mathrm{K}^{-} \mathrm{H}$ absorptions in the KLOE Drift Chamber [15] (DC) inner wall (aluminated carbon fiber), $\mathrm{K}^{-4} \mathrm{He}$ in the DC gas, filled with a mixture of Helium and Isobutane (in volume: 
$90 \%{ }^{4} \mathrm{He}$ and $10 \% \mathrm{C}_{4} \mathrm{H}_{10}$ ). Extremely rich experimental information is contained in this sample, with $\mathrm{K}^{-}$hadronic captures both at-rest and in-flight.

In order to increase the statistics and as an essential interpretation tool, a high purity carbon target (graphite) was realized in summer 2012 and installed inside the KLOE detector, between the beam pipe and the DC inner wall. The geometry of the target was optimized to maximize the kaon stopping power (technical details can be found in Ref. [13]). The total collected integrated luminosity is $\sim 90 \mathrm{pb}^{-1}$. Up to now we analysed a sample of $37 \mathrm{pb}^{-1}$ reconstructed data.

Details on the events selection and particle identification for the channels under investigation are given in Ref. [13].

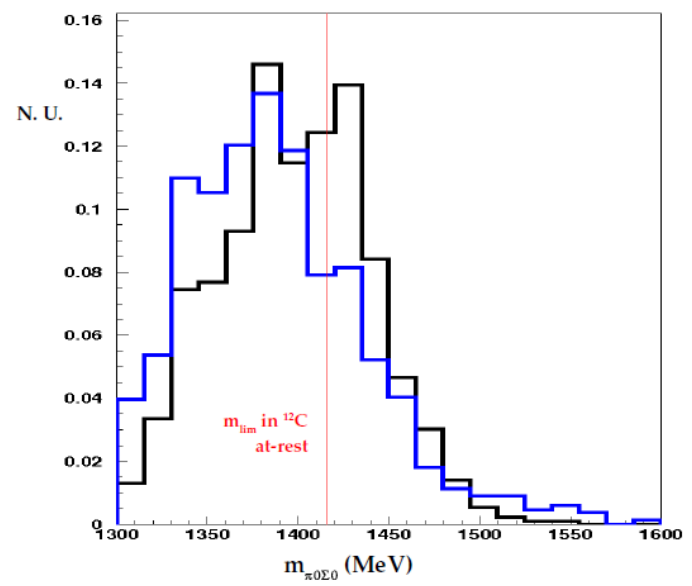

Figure 1. (Colour online.) $m_{\Sigma^{0} \pi^{0}}$ invariant mass distribution from $K^{-}$captures in the KLOE DC wall (black curve) and pure carbon graphite target (blue curve).

\section{Resonant and non resonant $\mathrm{Y}_{\pi}$ transition amplitudes below the $\bar{K} \mathbf{N}$ threshold}

The investigation of the $\Lambda(1405)$ properties in $\mathrm{K}^{-}$induced reactions on light nuclear targets is strongly biased by the kinematic threshold, determined by the absorbing nucleon binding energy. For $\mathrm{K}^{-}$captures at-rest on ${ }^{4} \mathrm{He}$ and ${ }^{12} \mathrm{C}$ the $m_{\Sigma \pi}$ invariant mass thresholds are about $1412 \mathrm{MeV}$ and $1416 \mathrm{MeV}$ respectively. The $\overline{\mathrm{K} N}$ sub-threshold region can be accessed in $\mathrm{K}^{-}$in-flight captures by exploiting the negative kaon kinetic energy contribution. For kaon momenta of $100 \mathrm{MeV} / \mathrm{c}$ the $m_{\Sigma \pi}$ threshold is shifted upwards of about $10 \mathrm{MeV}$, thus opening the mass window where the high mass pole is predicted.

In Fig. 1 (black distribution) the $\Sigma^{0} \pi^{0}$ invariant mass spectrum from $\mathrm{K}^{-}$captures in the KLOE DC wall is shown [16]. $\Sigma^{0} \pi^{0}$ represents the best signature of the $\Lambda(1405)$ resonance, since does not contain isospin $I=1$ background. The $m_{\Sigma 0 \pi 0}$ spectrum is compared with the corresponding distribution of $\mathrm{K}^{-}$captures at-rest in the pure Carbon target (fig. 1 blue distribution), the blue and the black distributions are normalised to unity. In fig. 1 a red line indicates the energy threshold corresponding to $\mathrm{K}^{-}$absorption in ${ }^{12} \mathrm{C}$ at-rest. A rich sample of in-flight $\mathrm{K}^{-12} \mathrm{C}$ captures can be easily identified above the red line. The $\Lambda(1405)$ shape can be now extracted after subtracting the $\Sigma^{0} \pi^{0}$ non-resonant contribution. 
In Ref. [17] and Ref. [18] the real and imaginary parts of the non-resonant coupled channels $\mathrm{K}^{-} \mathrm{n} \rightarrow \Lambda \pi / \Sigma \pi$ scattering amplitudes, calculated on the basis of several chiral SU(3) meson-baryon coupled channels interaction models (Prague (P) [19], Kyoto-Munich (KM) [6], Murcia (M1,M2) [20] Bonn (B2,B4) [21]) are compared. The amplitudes values strongly differ in the $\overline{\mathrm{K}} \mathrm{N}$ sub-threshold region depending on the adopted model.

In order to pin down the model to be adopted for the description of the observed $\Sigma^{0} \pi^{0}$ spectra, the $\mathrm{K}^{-} \mathrm{n} \rightarrow \Lambda \pi^{-}$transition was measured by exploiting $\mathrm{K}^{-} \mathrm{n}$ single nucleon absorptions in ${ }^{4} \mathrm{He}$. Since the $\Sigma^{-}(1385)$ (isospin $\left.I=1\right)$ resonance is well known, the corresponding non-resonant transition amplitude $\left(\left|A_{K^{-} n \rightarrow \Lambda \pi^{-}}\right|\right)$can be extracted, and used to test the chiral predictions below threshold. The details of the particle identification and events selection are given in Ref. [24]. The contribution of the $\Sigma$ particles conversion events to the selected sample was minimised by selecting, in the $p_{\pi^{-}}$vs $p_{\Lambda}$ scatterplot, the phase space region which is populated by the correlated direct $\Lambda \pi^{-}$production. The phase space cut was optimised based on MC simulations (performed with the standard KLOE GEANT digitization (GEANFI [22])), according to the calculations reported in [23].

In order to extract the ratio of the resonant over non-resonant $\Lambda \pi^{-}$production, the measured $p_{\Lambda \pi}, m_{\Lambda \pi}$ (invariant mass of the hyperon-pion pair) and $\cos \left(\theta_{\Lambda \pi}\right)\left(\theta_{\Lambda \pi}\right.$ is the angle between the $\Lambda$ and $\pi$ momenta) distributions were fitted. The main background is represented by the $\mathrm{K}^{-12} \mathrm{C}$ absorptions due to the Isobutane molecules in the DC gas. In order to account for this contribution an experimental sample of $\mathrm{K}^{-12} \mathrm{C}$ absorptions is used, obtained by selecting kaons interactions in the DC entrance wall. The Carbon-sample is selected adopting the same criteria as for the gas sample and was used in the global fit. The modulus of the amplitude of the non-resonant processes, the ratio of the resonant to non-resonant processes, the modulus of the amplitude of the conversions and the contribution of the $\mathrm{K}^{-}$captures on Carbon are considered as free parameters in the fit.

The results of the fit are summarised in Table I. (In Ref [24] the corresponding Fig. 3 is shown). The systematic errors are estimated by varying independently the selection cuts such as to increase or decrease the $\Lambda \pi^{-}$statistics by $15 \%$ with respect to the optimized selection. The chi-square of the fit is $\chi^{2} / n d f=151 / 148$. The ratio ( $\left.\frac{\mathrm{RES}-\mathrm{if}}{\mathrm{NR}-\mathrm{if}}\right)$ for the $\Lambda \pi^{-}$production in-flight is found to be smaller than the corresponding ratio at-rest. This is not surprising as the $\mathrm{K}^{-} \mathrm{n}$ interaction in-flight occurs about $\sqrt{s}=20 \mathrm{MeV}$ below the $\overline{\mathrm{K}} \mathrm{N}$ threshold; the corresponding reaction at-rest occurs about $\sqrt{s}=33 \mathrm{MeV}$ below the threshold (see Ref. [23]), nearer to the resonance which lays about $49 \mathrm{MeV}$ below the $\overline{\mathrm{K}} \mathrm{N}$ threshold. The systematic uncertainty on the resonant to non-resonant ratio for the in-flight reactions, prevents from extracting the modulus of the non-resonant transition amplitude in-flight. The corresponding transition amplitude modulus at-rest, extracted by comparing Eq. (14) and Eq. (20) in Ref. [23] with the experimental result $\left(\frac{\text { RES-ar }}{N R-a r}\right)$ is:

$$
\left|A_{K^{-} n \rightarrow \Lambda \pi^{-}}\right|=\left(0.334 \pm 0.018 \mathrm{stat}_{-0.058}^{+0.034} \mathrm{syst}\right) \mathrm{fm} .
$$

A similar analysis aiming to extract the BRs and cross sections of the $\mathrm{K}^{-}$multi-nucleon absorptions on two, three and four nucleons (2NA, 3NA and 4NA) is presently under finalisation. $\Lambda$ p correlated production events, originated in $\mathrm{K}^{-}$absorptions on ${ }^{12} \mathrm{C}$ are selected and a simultaneous fit of the $\Lambda \mathrm{p}$ invariant mass, angular correlation, $\Lambda$ and proton momenta is performed. Both the direct $\Lambda$ production and $\Sigma^{0}$ production (followed by $\Sigma^{0} \rightarrow \Lambda \gamma$ decay) are considered. The $\mathrm{K}^{-}$nuclear capture is modelled according to Refs. [23, 25]. Both elastic and inelastic final state interaction processes of the primary produced hyperons and protons are taken into account, as well as possible fragmentations of the residual nucleus. The possible contribution of $\mathrm{a} \mathrm{K}^{-} \mathrm{pp}$ bound state is also investigated, in analogy with the work described in Ref. [10]. 


\begin{tabular}{|l|l|l|l|}
\hline \hline Channels & Ratio/yield & $\sigma_{\text {stat }}$ & $\sigma_{\text {syst }}$ \\
\hline RES-ar/NR-ar & 0.39 & \pm 0.04 & ${ }_{-0.07}^{+0.18}$ \\
\hline RES-if/NR-if & 0.23 & \pm 0.03 & ${ }_{-0.22}^{+0.23}$ \\
\hline NR-ar & $12.0 \%$ & $\pm 1.7 \%$ & ${ }_{-2.8}^{+2.0} \%$ \\
\hline NR-if & $19.2 \%$ & $\pm 4.4 \%$ & ${ }_{-3.3}^{+5.9} \%$ \\
\hline$\Sigma \rightarrow \Lambda$ conv. & $2.2 \%$ & $\pm 0.3 \%$ & ${ }_{-0.8}^{+1.6} \%$ \\
\hline$K^{-12}$ C capture & $57.0 \%$ & $\pm 1.2 \%$ & ${ }_{-3.2}^{+2.2} \%$ \\
\hline \hline
\end{tabular}

Table 1. Resonant to non-resonant ratios and amplitudes of the various channels extracted from the fit of the $\Lambda \pi^{-}$sample. The statistical and systematic errors are also shown. See text for details.

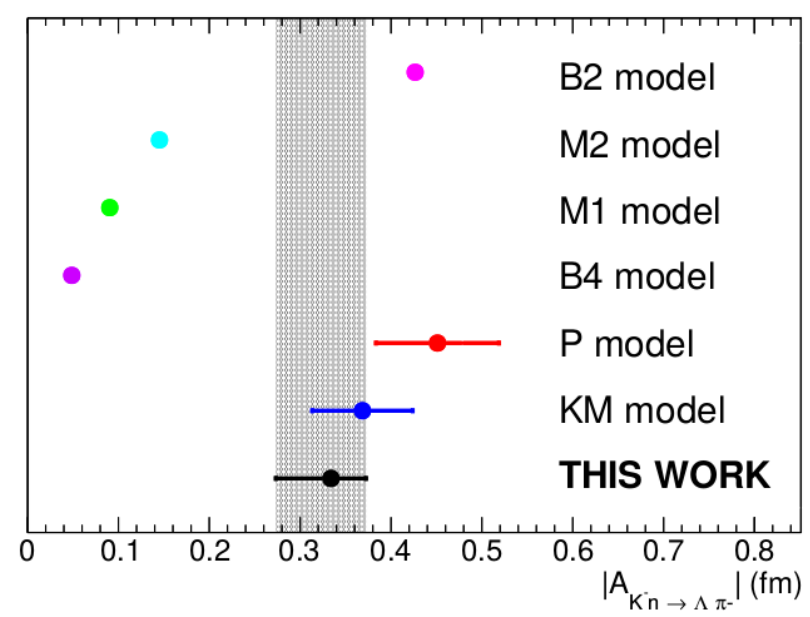

Figure 2. (Color online) Modulus of the measured non resonant $K^{-} n \rightarrow \Lambda \pi^{-}$transition amplitude compared with theoretical calculations, see details in the text.

\section{Discussion}

In this work the measurement of the isospin $I=1$ non-resonant transition amplitude $\left|A_{K^{-} n \rightarrow \Lambda \pi^{-}}\right|$at $\sqrt{s}=33 \mathrm{MeV}$ below the $\overline{\mathrm{K}} \mathrm{N}$ threshold is presented. The result of this analysis (with combined statistical and systematic errors) is shown in Fig. 2 and compared with the theoretical predictions (see Ref: [19] (P), [6] (KM), [20] (M1,M2), [21] (B2,B4)), rescaled for the $\mathrm{K}^{-} \mathrm{n} \rightarrow \Sigma \pi$ transition probabilities. The presented result allows an extrapolation to the un-physical region and can be used to test models of S-wave interaction; moreover such extrapolation can be used to constrain the corresponding $(\Sigma \pi)^{0}$ non-resonant background for the extraction of the $\Lambda(1405)$ properties in $\overline{\mathrm{K}} \mathrm{N}$ absorption experiments.

\section{Acknowledgments}

We acknowledge the KLOE/KLOE-2 Collaboration for their support and for having provided us the data and the tools to perform the analysis presented in this paper. We acknowledge the 
CENTRO FERMI - Museo Storico della Fisica e Centro Studi e Ricerche "Enrico Fermi", for the project PAMQ. Part of this work was supported by the Austrian Science Fund (FWF): [P24756-N20]; Austrian Federal Ministry of Science and Research BMBWK 650962/0001 VI/2/2009; the Croatian Science Foundation, under project 1680; Minstero degli Affari Esteri e della Cooperazione Internazionale, Direzione Generale per la Promozione del Sistema Paese (MAECI), Strange Matter project; Polish National Science Center through grant No. UMO-2016/21/D/ST2/01155.

\section{References}

[1] A. Gallo et al., Conf. Proc. C 060626, 604 (2006)

[2] J. A. Oller and U. G. Meissner, Phys. Lett. B 500, 263 (2001)

[3] T. Hyodo et al., Phys. Rev. C 68, 018201 (2003)

[4] D. Jido et al., Nucl. Phys. A 725, 181 (2003)

[5] T. Hyodo and D. Jido, Prog. Part. Nucl. Phys. 67, 55 (2012)

[6] Y. Ikeda, T. Hyodo and W. Weise, Nucl. Phys. A 881, 98 (2012)

[7] V. K. Magas, E. Oset, A. Ramos and H. Toki, Phys. Rev. C 74, 025206 (2006)

[8] V. K. Magas, E. Oset and A. Ramos, Phys. Rev. C 77, 065210 (2008)

[9] T. Suzuki et al. [KEK-PS E549 Collaboration], Mod. Phys. Lett. A 23, 2520 (2008)

[10] O. Vazquez Doce et al., Phys. Lett. B 758, 134 (2016)

[11] V. Metag, M. Nanova and E. Ya. Paryev, Prog. Part. Nucl. Phys. 97, 199 (2017)

[12] F. Bossi, E. De Lucia, J. Lee-Franzini, S. Miscetti, M. Palutan [KLOE Collaboration], Riv. Nuovo Cim. 31, 531 (2008)

[13] C. Curceanu et al., Acta Phys. Polon. B 46, 203 (2015)

[14] O. Vazquez Doce et al., Phys. Lett. B 758, 134 (2016)

[15] M. Adinolfi et al., Nucl. Instrum. Meth. A 488, 51 (2002)

[16] K. Piscicchia, $\mathrm{PhD}$ thesis (2013), Università degli Studi di Roma Tre, http://www.infn.it/thesis/PDF/getfile.php?filename=7097-Piscicchia-dottorato.pdf

[17] J. Hrtankova and J. Mares, Phys. Rev. C 96, 015205 (2017)

[18] A. Cieplý et al., Nucl. Phys. A 954, 17 (2016)

[19] A. Cieplý and J. Smejkal, Nucl. Phys. A 881, 115 (2012)

[20] Z. H. Guo and J. A. Oller, Phys. Rev. C87, 035202 (2013)

[21] M. Mai and U. G. Meißner, Eur. Phys. J. A 51, 30 (2015)

[22] F. Ambrosino et al., Nucl. Instrum. Meth. A 534, 403 (2004)

[23] K. Piscicchia, S. Wycech and C. Curceanu, Nucl. Phys. A 954, 75 (2016)

[24] K. Piscicchia et al., Phys. Lett. B 782, 339 (2018)

[25] R. Del Grande, K. Piscicchia and S. Wycech, Acta Phys. Polon. B 48, 1881 (2017) 